PROCEEDINGS OF THE

AMERICAN MATHEMATICAL SOCIETY

Volume 137, Number 8, August 2009, Pages 2517-2528

S 0002-9939(09)09886-4

Article electronically published on March 17, 2009

\title{
A MULTIPLICATION FORMULA FOR MODULE SUBCATEGORIES OF EXT-SYMMETRY
}

\author{
JIE XIAO AND FAN XU
}

(Communicated by Martin Lorenz)

\begin{abstract}
We define evaluation forms associated to objects in a module subcategory of Ext-symmetry generated by finitely many simple modules over a path algebra with relations and prove a multiplication formula for the product of two evaluation forms. It is analogous to a multiplication formula for the product of two evaluation forms associated to modules over a preprojective algebra given by Geiss, Leclerc and Schröer in Compositio Math. 143 (2007), $1313-1334$.
\end{abstract}

\section{INTRODUCTION}

Let $\Lambda$ be the preprojective algebra associated to a connected quiver without loops (see e.g. [12]) and let $\bmod (\Lambda)$ be the category of finite-dimensional nilpotent left $\Lambda$ modules. We denote by $\Lambda_{\underline{e}}$ the variety of finite-dimensional nilpotent left $\Lambda$-modules with dimension vector $\underline{e}$. For any $x \in \Lambda_{\underline{e}}$, there is an evaluation form $\delta_{x}$ associated to $x$ satisfying that there is a finite subset $R(\underline{e})$ of $\Lambda_{\underline{e}}$ such that $\Lambda_{\underline{e}}=\bigsqcup_{x \in R(\underline{e})}\langle x\rangle$, where $\langle x\rangle:=\left\{y \in \Lambda_{\underline{e}} \mid \delta_{x}=\delta_{y}\right\}$ [7, Section 1.2]. Inspired by the Caldero-Keller cluster multiplication theorem for finite type [4, Geiss, Leclerc and Schröer [7] proved a multiplication formula (the Geiss-Leclerc-Schröer multiplication formula) as follows:

$$
\chi\left(\mathbb{P E x t}_{\Lambda}^{1}\left(x^{\prime}, x^{\prime \prime}\right)\right) \delta_{x^{\prime} \oplus x^{\prime \prime}}=\sum_{x \in R(\underline{e})}\left(\chi\left(\mathbb{P E x t}_{\Lambda}^{1}\left(x^{\prime}, x^{\prime \prime}\right)_{\langle x\rangle}\right)+\chi\left(\operatorname{PExt}_{\Lambda}^{1}\left(x^{\prime \prime}, x^{\prime}\right)_{\langle x\rangle}\right)\right) \delta_{x},
$$

where $x^{\prime} \in \Lambda_{\underline{e}^{\prime}}, x^{\prime \prime} \in \Lambda_{\underline{e}^{\prime \prime}}, \underline{e}=\underline{e}^{\prime}+\underline{e}^{\prime \prime}, \mathbb{P E x t}{ }_{\Lambda}^{1}\left(x^{\prime}, x^{\prime \prime}\right)_{\langle x\rangle}$ is the constructible subset of $\operatorname{PExt}_{\Lambda}^{1}\left(x^{\prime}, x^{\prime \prime}\right)$ with the middle terms belonging to $\langle x\rangle$, and $\operatorname{PExt}_{\Lambda}^{1}\left(x^{\prime \prime}, x^{\prime}\right)_{\langle x\rangle}$ is defined similarly.

The proof of the formula depends heavily on the fact that the category $\bmod (\Lambda)$ is of Ext-symmetry. A category $\mathcal{C}$ is of Ext-symmetry if there is a bifunctorial isomorphism: $\operatorname{Ext}_{\mathcal{C}}^{1}(M, N) \cong \operatorname{DExt}_{\mathcal{C}}^{1}(N, M)$ for any objects $M, N \in \mathcal{C}$.

Let $Q$ be a finite quiver and $A$ be a quotient algebra $\mathbb{C} Q / \mathcal{I}$ by an ideal $\mathcal{I}$. We denote by $\bmod (A)$ the category of finite-dimensional left $A$-modules. We call $A$ an algebra of Ext-symmetry if $\bmod (A)$ is of Ext-symmetry. It is proved that preprojective algebras and deformed preprojective algebras are of Ext-symmetry (see [7, Theorem 3] and Section 3 in this paper).

Received by the editors January 18, 2008, and, in revised form, September 26, 2008. 2000 Mathematics Subject Classification. Primary 16G20, 14M99; Secondary 20G05.

Key words and phrases. Ext-symmetry, module variety, flag variety, composition series. The research was supported in part by NSF of China (No. 10631010).

(C)2009 American Mathematical Society 2517

Reverts to public domain 28 years from publication 
In this paper, we focus on the module subcategories of Ext-symmetry of $\bmod (A)$. Let $\mathcal{S}=\left\{S_{1}, \cdots, S_{n}\right\}$ be a finite subset of finite-dimensional simple $A$-modules. We denote by $\mathcal{C}(\mathcal{S})$ the full subcategory of $\bmod (A)$ consisting of modules $M$ satisfying that the isomorphism classes of the composition factors of $M$ belong to $\mathcal{S}$. We associate to modules in $\mathcal{C}(\mathcal{S})$ some evaluation forms and prove that if $\mathcal{C}(\mathcal{S})$ is of Extsymmetry, then the product of two evaluation forms satisfies an identity (Theorem 2.3). The identity is analogous to the Geiss-Leclerc-Schröer multiplication formula. There are no known examples of algebras of Ext-symmetry, apart from preprojective and deformed preprojective algebras (see Section 3), and it is an open question whether further examples exist. However, other examples of module subcategories of Ext-symmetry can be easily constructed, and we give an example in Section 3.

\section{The PRODUCT OF TWO EVALUATION FORMS}

1.1. Module varieties. Let $Q=\left(Q_{0}, Q_{1}, s, t\right)$ be a finite connected quiver, where $Q_{0}$ and $Q_{1}$ are the sets of vertices and arrows, respectively, and $s, t: Q_{1} \rightarrow Q_{0}$ are maps such that any arrow $\alpha$ starts at $s(\alpha)$ and terminates at $t(\alpha)$. The space spanned by all paths of nonzero length is a graded ideal of $\mathbb{C} Q$, and we will denote it by $\mathcal{J}$. A relation for $Q$ is a linear combination $\sum_{i=1}^{r} \lambda_{i} p_{i}$, where $\lambda_{i} \in \mathbb{C}$ and the $p_{i}$ are paths with $s\left(p_{i}\right)=s\left(p_{j}\right)$ and $t\left(p_{i}\right)=t\left(p_{j}\right)$ for any $1 \leq i, j \leq r$. Here if $p_{i}$ is a vertex in $Q_{0}$, then $s\left(p_{i}\right)=t\left(p_{i}\right)=p_{i}$. Let $A=\mathbb{C} Q / \mathcal{I}$, where $\mathcal{I}$ is an ideal generated by a finite set of relations. We don't assume that $\mathcal{I}$ is admissible, i.e. $\mathcal{I} \subset \mathcal{J}^{2}$.

A dimension vector for $A$ is a map $\underline{d}: Q_{0} \rightarrow \mathbb{N}$. We write $d_{i}$ instead of $d(i)$ for any $i \in Q_{0}$. For any dimension vector $\underline{d}=\left(d_{i}\right)_{i \in Q_{0}}$, we consider the affine space over $\mathbb{C}$,

$$
\mathbb{E}_{\underline{d}}(Q)=\bigoplus_{\alpha \in Q_{1}} \operatorname{Hom}_{\mathbb{C}}\left(\mathbb{C}^{d_{s(\alpha)}}, \mathbb{C}^{d_{t(\alpha)}}\right)
$$

Any element $x=\left(x_{\alpha}\right)_{\alpha \in Q_{1}}$ in $\mathbb{E}_{\underline{d}}(Q)$ defines a representation $\left(\mathbb{C}^{\underline{d}}, x\right)$, where $\mathbb{C}^{\underline{d}}=$ $\bigoplus_{i \in Q_{0}} \mathbb{C}^{d_{i}}$. For any $x=\left(x_{\alpha}\right)_{\alpha \in Q_{1}} \in \mathbb{E}_{\underline{d}}(Q)$ and any path $p=\alpha_{1} \alpha_{2} \cdots \alpha_{m}$ in $Q$, we set $x_{p}=x_{\alpha_{1}} x_{\alpha_{2}} \cdots x_{\alpha_{m}}$. Then $x$ satisfies a relation $\sum_{i=1}^{r} \lambda_{i} p_{i}$ if $\sum_{i=1}^{r} \lambda_{i} x_{p_{i}}=0$. Here if $p_{i}$ is a vertex in $Q_{0}$, then $x_{p_{i}}$ is the identity matrix. Let $R$ be a finite set of relations generating the ideal $\mathcal{I}$. Then we denote by $\mathbb{E}_{\underline{d}}(A)$ the closed subvariety of $\mathbb{E}_{\underline{d}}(Q)$ which consists of elements satisfying all relations in $R$.

Let $\mathcal{S}=\left\{S_{1}, \cdots, S_{n}\right\}$ be a finite subset of finite-dimensional simple $A$-modules and $\mathcal{C}(\mathcal{S})$ be a module subcategory of Ext-symmetry of $\bmod (A)$. We denote by $A_{\underline{d}}(\mathcal{S})$ the constructible subset of $\mathbb{E}_{\underline{\underline{d}}}(A)$ consisting of modules in $\mathcal{C}(\mathcal{S})$. In the sequel, we will fix the finite set $\mathcal{S}$ and write $A_{\underline{\underline{d}}}$ instead of $A_{\underline{\underline{d}}}(\mathcal{S})$. The algebraic group $G_{\underline{d}}:=G_{\underline{d}}(Q)=\prod_{i \in Q_{0}} \mathrm{GL}_{d_{i}}(\mathbb{C})$ acts on $\mathbb{E}_{\underline{d}}(Q)$ by $\left(x_{\alpha}\right)_{\alpha \in Q_{1}}^{g}=\left(g_{t(\alpha)} x_{\alpha} g_{s(\alpha)}^{-1}\right)_{\alpha \in Q_{1}}$ for $g \in G_{\underline{d}}$ and $\left(x_{\alpha}\right)_{\alpha \in Q_{1}} \in \mathbb{E}_{\underline{\underline{d}}}(Q)$. It naturally induces the action of $G_{\underline{d}}$ on $A_{\underline{d}}(\mathcal{S})$. The orbit space is denoted by $\bar{A}_{\underline{d}}(\mathcal{S})$. A constructible function over $\mathbb{E}_{\underline{d}}(A)$ is a function $f: \mathbb{E}_{\underline{d}}(A) \rightarrow \mathbb{C}$ such that $f\left(\mathbb{E}_{\underline{d}}(A)\right)$ is a finite subset of $\mathbb{C}$ and $f^{-1}(c)$ is a constructible subset of $\mathbb{E}_{\underline{d}}(A)$ for any $c \in Q$.

Throughout this paper, we always assume that $\mathcal{C}(\mathcal{S})$ is of Ext-symmetry and that constructible functions over $\mathbb{E}_{\underline{d}}(A)$ are $G_{\underline{\underline{d}}}$-invariant for any dimension vector $\underline{d}$ unless particularly stated.

1.2. Euler characteristics. Let $\chi$ denote the Euler characteristic in compactly supported cohomology. Let $X$ be a complex algebraic variety and $\mathcal{O}$ a constructible 
subset as the disjoint union of finitely many locally closed subsets $X_{i}$ for $i=$ $1, \cdots, m$. Define $\chi(\mathcal{O})=\sum_{i=1}^{m} \chi\left(X_{i}\right)$. We note that it is well-defined. The following properties will be applied to compute Euler characteristics.

Proposition 1.1 ([1] and [9]). Let $X, Y$ be algebraic varieties over $\mathbb{C}$. Then

(1) If an algebraic variety $X$ is the disjoint union of finitely many constructible sets $X_{1}, \cdots, X_{r}$, then

$$
\chi(X)=\sum_{i=1}^{r} \chi\left(X_{i}\right)
$$

(2) If $\varphi: X \longrightarrow Y$ is a morphism with the property that all fibers have the same Euler characteristic $\chi$, then $\chi(X)=\chi \cdot \chi(Y)$. In particular, if $\varphi$ is a locally trivial fibration in the analytic topology with fibre $F$, then $\chi(Z)=$ $\chi(F) \cdot \chi(Y)$.

(3) $\chi\left(\mathbb{C}^{n}\right)=1$ and $\chi\left(\mathbb{P}^{n}\right)=n+1$ for all $n \geq 0$.

We recall the pushforward functor from the category of algebraic varieties over $\mathbb{C}$ and the category of $\mathbb{C}$-vector spaces (see [10] and [9]). Let $\phi: X \rightarrow Y$ be a morphism of varieties. Write $M(X)$ for the $\mathbb{C}$-vector space of constructible functions on $X$. For $f \in M(X)$ and $y \in Y$, define

$$
\phi_{*}(f)(y)=\sum_{c \neq 0} c \chi\left(f^{-1}(c) \cap \phi^{-1}(y)\right) .
$$

Theorem $1.2([5,[9])$. Let $X, Y$ and $Z$ be algebraic varieties over $\mathbb{C}, \phi: X \rightarrow$ $Y$ and $\psi: Y \rightarrow Z$ be morphisms of varieties, and $f \in M(X)$. Then $\phi_{*}(f)$ is constructible, $\phi_{*}: M(X) \rightarrow M(Y)$ is a $\mathbb{C}$-linear map and $(\psi \circ \phi)_{*}=(\psi)_{*} \circ(\phi)_{*}$ as $\mathbb{C}$-linear maps from $M(X)$ to $M(Z)$.

1.3. The actions of $\mathbb{C}^{*}$ on the extensions and flags. Let $A=\mathbb{C} Q /\langle R\rangle$ be an algebra as in Section 1.1. For any $A$-modules $X, Y$, let $D(X, Y)$ be the vector space over $\mathbb{C}$ of all tuples $d=(d(\alpha))_{\alpha \in Q_{1}}$ such that linear maps $d(\alpha) \in \operatorname{Hom}_{\mathbb{C}}\left(X_{s(\alpha)}, Y_{t(\alpha)}\right)$ and the matrices $L(d)_{\alpha}=\left(\begin{array}{cc}Y_{\alpha} & d(\alpha) \\ 0 & X_{\alpha}\end{array}\right)$ satisfy the relations in $R$. Define $\pi$ : $D(X, Y) \rightarrow \operatorname{Ext}^{1}(X, Y)$ by sending $d$ to the equivalence class of the following short exact sequence:

$$
\varepsilon: \quad 0 \longrightarrow Y \stackrel{\left(\begin{array}{l}
1 \\
0
\end{array}\right)}{\longrightarrow} L(d) \stackrel{\left(\begin{array}{ll}
0 & 1
\end{array}\right)}{\longrightarrow} X \longrightarrow 0
$$

where, as a vector space, $L(d)=\left(L(d)_{\alpha}\right)_{\alpha \in Q_{1}}$ is the direct sum of $Y$ and $X$. The direct computation shows that $\operatorname{Ker} \pi$ is the subspace of $D(X, Y)$ consisting of all tuples $d=(d(\alpha))_{\alpha \in Q_{1}}$ such that there exist $\left(\phi_{i}\right)_{i \in Q_{0}} \in \bigoplus_{i \in Q_{0}} \operatorname{Hom}_{\mathbb{C}}\left(X_{i}, Y_{i}\right)$ satisfying $d(\alpha)=\phi_{t(\alpha)} X_{\alpha}-Y_{\alpha} \phi_{s(\alpha)}$ for all $\alpha \in Q_{1}$ (see [7, Section 5.1] for a similar discussion).

Fix a vector space decomposition $D(X, Y)=\operatorname{Ker} \pi \oplus E(X, Y)$. We can identify $\operatorname{Ext}_{A}^{1}(X, Y)$ with $E(X, Y)\left([11,[6],[7])\right.$. Let $\operatorname{Ext}_{A}^{1}(X, Y)_{L}$ be the subset of $\operatorname{Ext}_{A}^{1}(X, Y)$ with the middle term isomorphic to $L$. Then $\operatorname{Ext}^{1}(X, Y)_{L}$ can be viewed as a constructible subset of $\operatorname{Ext}_{A}^{1}(X, Y)$ by the identification between 
$\operatorname{Ext}_{A}^{1}(X, Y)$ and $E(X, Y)$. There is a natural $\mathbb{C}^{*}$-action on $E(X, Y) \backslash\{0\}$ by $t . d=$ $(t d(\alpha))$ for any $t \in \mathbb{C}^{*}$. This induces the action of $\mathbb{C}^{*}$ on $\operatorname{Ext}_{A}^{1}(X, Y) \backslash\{0\}$. For any $t \in \mathbb{C}^{*}$, we have that $t . \varepsilon$ is the following short exact sequence:

$$
0 \longrightarrow Y \stackrel{\left(\begin{array}{l}
1 \\
0
\end{array}\right)}{\longrightarrow} L(t . d) \stackrel{\left(\begin{array}{ll}
0 & 1
\end{array}\right)}{\longrightarrow} X \longrightarrow 0
$$

where $L(t . d)_{\alpha}=\left(\begin{array}{cc}Y_{\alpha} & t d(\alpha) \\ 0 & X_{\alpha}\end{array}\right)$ for any $\alpha \in Q_{1}$. The orbit space is denoted by $\mathbb{P E x t}{ }_{A}^{1}(X, Y)$ and the orbit of $\varepsilon \in \operatorname{Ext}_{A}^{1}(X, Y)$ is denoted by $\mathbb{P} \varepsilon$. For a $G_{\underline{d}}$-invariant constructible subset $\mathcal{O}$ of $\mathbb{E}_{\underline{d}}(A)$, we set $\operatorname{Ext}_{A}^{1}(X, Y)_{\mathcal{O}}$ to be the subset of $\operatorname{Ext}_{A}^{1}(X, Y)$ consisting of the equivalence classes of extensions with middle terms belonging to $\mathcal{O}$.

The above $\mathbb{C}^{*}$-action on the extensions induces an action on the middle terms. As a vector space, $L=Y \oplus X$. So we can define $t .(y, x)=(t y, x)$ for any $t \in \mathbb{C}^{*}$ and $x \in X, y \in Y$ [7, Section 5.4] or [11, Lemma 1]. For any $L_{1} \subseteq L$, this action yields a submodule $t . L_{1}$ of $L$ isomorphic to $L_{1}$. In general, if $\mathfrak{f}_{L}=\left(L \supseteq L_{1} \supseteq L_{2} \supseteq\right.$ $\left.\cdots \supseteq L_{m}=0\right)$ is a flag of submodules of $L$, then $t \cdot f_{L}=\left(L \supseteq t . L_{1} \supseteq t . L_{2} \supseteq \cdots \supseteq\right.$ t. $\left.L_{m}=0\right)$. Hence, we obtain an action of $\mathbb{C}^{*}$ on the flag of $L$.

1.4. The product of two evaluation forms. Let $A_{\underline{d}}:=A_{\underline{d}}(\mathcal{S})$ be the constructible subset of $\mathbb{E}_{\underline{d}}(A)$ as in Section 1.1. For any module $M \in \mathbb{E}_{\underline{d}}(A)$, let $G r_{\underline{\underline{e}}}(M)$ be the subvariety of $G r_{\underline{e}}\left(\mathbb{C}^{\underline{d}}\right):=\prod_{i \in Q_{0}} G r_{e_{i}}\left(\mathbb{C}^{d_{i}}\right)$ consisting of submodules of $M$ with dimension vector $\underline{e}=\left(e_{i}\right)_{i \in Q_{0}}$, and let $G r_{\underline{e}}\left(\mathbb{E}_{\underline{d}}(A)\right)$ be the constructible subset of $\mathbb{E}_{\underline{d}}(A) \times G r_{\underline{e}}(\mathbb{C} \underline{d})$ consisting of pairs $\left(M, M_{1}\right)$ such that $M_{1} \in G r_{\underline{e}}(M)$.

Proposition 1.3. Let $\underline{d}$ and $\underline{e}$ be two dimension vectors. Then the function $\operatorname{gr}(\underline{e}, \underline{d}): \mathbb{E}_{\underline{d}}(A) \rightarrow \mathbb{C}$ sending $M$ to $\chi\left(G r_{\underline{e}}(M)\right)$ is a $G_{\underline{\underline{d}}}$-invariant constructible function.

Proof. Consider the projection: $\phi: G r_{\underline{e}}\left(\mathbb{E}_{\underline{d}}(A)\right) \rightarrow \mathbb{E}_{\underline{d}}(A)$ mapping $\left(M, M_{1}\right)$ to $M$. It is clear that $\phi$ is a morphism of varieties. By Theorem $1.2, g r(\underline{e}, \underline{d})=$ $\phi_{*}\left(1_{G r_{\underline{e}}\left(\mathbb{E}_{\underline{d}}(A)\right)}\right)$ is constructible.

For fixed $\underline{d}$, we can make finitely many choices of $\underline{e}$ such that $G r_{\underline{e}}\left(\mathbb{E}_{\underline{d}}(A)\right)$ is nonempty. This implies the following corollary.

Corollary 1.4. There is a finite subset $S(\underline{d})$ of $A_{\underline{d}}$ such that $A_{\underline{d}}=\bigcup_{i \in S(\underline{d})} \mathcal{O}(\underline{d})_{i}$, where all $\mathcal{O}(\underline{d})_{i}$ are constructible subsets of $A_{\underline{d}}$ satisfying that for any $M, M^{\prime} \in$ $\mathcal{O}(\underline{d})_{i}, \chi\left(G r_{\underline{e}}(M)\right)=\chi\left(G r_{\underline{e}}\left(M^{\prime}\right)\right)$ for any $\underline{e}$.

Let $\mathcal{M}(\underline{d})$ be the vector space over $\mathbb{C}$ spanned by the constructible functions $\operatorname{gr}(\underline{e}, \underline{d})$ for any dimension vector $\underline{e}$. For any $M \in A_{\underline{d}}$, we define the evaluation form $\delta_{M}: \mathcal{M}(\underline{d}) \rightarrow \mathbb{C}$ which maps the constructible function $\operatorname{gr}(\underline{e})$ to $\chi\left(G r_{\underline{e}}(M)\right)=$ $\operatorname{gr}(\underline{e})(M)$. Using the notation in [7], we set $\langle L\rangle:=\mathcal{O}(\underline{d})_{i}$ for arbitrary $L \in \mathcal{O}(\underline{d})_{i}$. Indeed, $\delta_{L}=\delta_{L^{\prime}}$ for any $L, L^{\prime} \in \mathcal{O}(\underline{d})_{i}$. By abuse of notation, we have $A_{\underline{d}}=$ $\bigcup_{L \in S(\underline{d})}\langle L\rangle$.

Let $M, N$ be $A$-modules and $\underline{e}_{1}, \underline{e}_{2}$ be dimension vectors. Fixing $M_{1} \in G r_{\underline{e}_{1}}(M)$, $N_{1} \in G r_{\underline{e}_{2}}(N)$, we consider the natural map

$$
\beta_{N_{1}, M_{1}}: \operatorname{Ext}_{A}^{1}\left(N, M_{1}\right) \rightarrow \operatorname{Ext}_{A}^{1}(N, M) \oplus \operatorname{Ext}_{A}^{1}\left(N_{1}, M_{1}\right)
$$


mapping $\varepsilon_{*} \in \operatorname{Ext}_{A}^{1}\left(N, M_{1}\right)$ to $\left(\varepsilon, \varepsilon^{\prime}\right)$ such that the following diagram commutes:

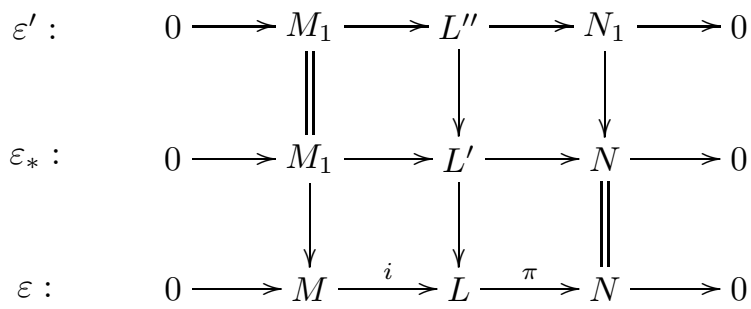

where $L$ and $L^{\prime \prime}$ are the pushout and pullback, respectively. Define

$$
\begin{aligned}
& E F_{\underline{\underline{1}}_{1}, \underline{e}_{2}}^{g}(N, M)=\left\{\left(M_{1}, N_{1}, \varepsilon, L_{1}\right) \mid M_{1} \in G r_{\underline{e}_{1}}(M), N_{1} \in G r_{\underline{e}_{2}}(N), \varepsilon \neq 0 \in\right. \\
& \left.\operatorname{Ext}_{A}^{1}(N, M)_{L} \cap \operatorname{Im} \beta_{N_{1}, M_{1}}, L_{1} \in G r_{\underline{e}_{1}+\underline{e}_{2}}(L), L_{1} \cap i(M)=i\left(M_{1}\right), \pi\left(L_{1}\right)=N_{1}\right\}
\end{aligned}
$$

and $E F_{\underline{e}}^{g}(N, M)=\bigcup_{\underline{e}_{1}+\underline{e}_{2}=\underline{e}} E F_{\underline{e}_{1}, \underline{e}_{2}}^{g}(N, M)$. By the discussion in Section 1.3, the action of $\mathbb{C}^{*}$ on $\operatorname{Ext}_{A}^{1}(N, M) \backslash\{0\}$ naturally induces the action on $E F_{\underline{e}}^{g}(N, M)$ by setting

$$
\text { t. }\left(M_{1}, N_{1}, \varepsilon, L_{1}\right)=\left(M_{1}, N_{1}, t . \varepsilon, t . L_{1}\right)
$$

for $\left(M_{1}, N_{1}, \varepsilon, L_{1}\right) \in E F_{\underline{e}}^{g}(N, M)$ and $t \in \mathbb{C}^{*}$. We denote its orbit space by $\mathbb{P} E F_{e}^{g}(N, M)$. We also set the evaluation form $\delta: \mathcal{M} \rightarrow \mathbb{C}$ mapping $g r(\underline{e})$ to $\chi\left(\mathbb{P} \underline{E} F_{\underline{e}}^{g}(N, M)\right)$.

Theorem 1.5. Let $M, N \in \mathcal{C}(\mathcal{S})$. We have

$$
\chi\left(\operatorname{PExt}_{A}^{1}(M, N)\right) \delta_{M \oplus N}=\sum_{L \in S(\underline{d})} \chi\left(\mathbb{P E x t}_{A}^{1}(M, N)_{\langle L\rangle}\right) \delta_{L}+\delta .
$$

Proof. Since (for example, see [1] or [6])

$$
\chi\left(G r_{\underline{e}}(M \oplus N)\right)=\sum_{\underline{e}_{1}+\underline{e}_{2}=\underline{e}} \chi\left(G r_{\underline{e}_{1}}(M)\right) \cdot \chi\left(G r_{\underline{e}_{2}}(N)\right),
$$

the above formula has the following reformulation:

$$
\begin{aligned}
\chi( & \left.\mathbb{P E x t}{ }_{A}^{1}(M, N)\right) \sum_{\underline{e}_{1}+\underline{e}_{2}=\underline{e}} \chi\left(G r_{\underline{e}_{1}}(M)\right) \cdot \chi\left(G r_{\underline{e}_{2}}(N)\right) \\
& =\sum_{L \in S(\underline{d})} \chi\left(\mathbb{P E x t}{ }_{A}^{1}(M, N)_{\langle L\rangle}\right) \chi\left(G r_{\underline{e}}(L)\right)+\chi\left(\mathbb{P} E F_{\underline{e}}^{g}(N, M)\right) .
\end{aligned}
$$

Now we prove the above reformulation. Define

$$
E F(M, N)=\left\{\left(\varepsilon, L_{1}\right) \mid \varepsilon \in \operatorname{Ext}_{A}^{1}(M, N)_{L} \backslash\{0\}, L_{1} \in G r_{\underline{e}}(L)\right\} .
$$

The action of $\mathbb{C}^{*}$ on $\operatorname{Ext}_{A}^{1}(M, N)$ naturally induces the action on $\operatorname{EF}(M, N)$ [7, section 5.4 ]. Under the action of $\mathbb{C}^{*}$, it has the geometric quotient:

$$
\pi: \operatorname{EF}(M, N) \rightarrow \mathbb{P} E F(M, N) .
$$

We have the natural projection:

$$
p: \mathbb{P E F}(M, N) \rightarrow \operatorname{PExt}_{A}^{1}(M, N) .
$$

Using Proposition 1.1, we have

$$
\chi(\mathbb{P} E F(M, N))=\sum_{L \in S(\underline{d})} \chi\left(\mathbb{P E x t}{ }_{A}^{1}(M, N)_{\langle L\rangle}\right) \chi\left(G r_{\underline{e}}(L)\right) .
$$


Given $\left(\varepsilon, L_{1}\right) \in \operatorname{EF}(M, N)$, let $\varepsilon$ be the equivalence class of the following short exact sequence:

$$
\varepsilon: \quad 0 \longrightarrow N \stackrel{\left(\begin{array}{l}
1 \\
0
\end{array}\right)}{\longrightarrow} L \stackrel{\left(\begin{array}{ll}
0 & 1
\end{array}\right)}{\longrightarrow} M \longrightarrow 0 .
$$

As a vector space, $L=N \oplus M$ and $L_{1}$ is the subspace of $L$. We put $M_{1}=(0,1)\left(L_{1}\right)$ and $N_{1}=(1,0)\left(L_{1}\right)$. It is clear that $M_{1}$ and $N_{1}$ are the submodules of $M$ and $N$, respectively. Then there is a natural morphism

$$
\phi_{0}: E F(M, N) \rightarrow \bigcup_{\underline{e}_{1}+\underline{e}_{2}=\underline{e}} G r_{\underline{e}_{1}}(M) \times G r_{\underline{e}_{2}}(N)
$$

defined by mapping $\left(\varepsilon, L_{1}\right)$ to $\left(M_{1}, N_{1}\right)$. Furthermore, we have

$$
\phi_{0}\left(\left(\varepsilon, L_{1}\right)\right)=\phi_{0}\left(t .\left(\varepsilon, L_{1}\right)\right)
$$

for any $\left(\varepsilon, L_{1}\right) \in E F(M, N)$ and $t \in \mathbb{C}^{*}$. This induces the morphism

$$
\phi: \mathbb{P} E F(M, N) \rightarrow \bigcup_{\underline{e}_{1}+\underline{e}_{2}=\underline{e}} G r_{\underline{e}_{1}}(M) \times G r_{\underline{e}_{2}}(N) .
$$

Now we compute the fibre of this morphism for $M_{1} \in G r_{\underline{e}_{1}}(M)$ and $N_{1} \in G r_{\underline{e}_{2}}(N)$. Consider the following linear map dual to $\beta_{M_{1}, N_{1}}$ :

$$
\beta_{M_{1}, N_{1}}^{\prime}: \operatorname{Ext}_{A}^{1}(M, N) \oplus \operatorname{Ext}_{A}^{1}\left(M_{1}, N_{1}\right) \rightarrow \operatorname{Ext}^{1}\left(M_{1}, N\right)
$$

mapping $\left(\varepsilon, \varepsilon^{\prime}\right)$ to $\varepsilon_{M_{1}}-\varepsilon_{N}^{\prime}$, where $\varepsilon_{M_{1}}$ and $\varepsilon_{N}^{\prime}$ are induced by the inclusions $M_{1} \subseteq M$ and $N_{1} \subseteq N$, respectively, as follows:

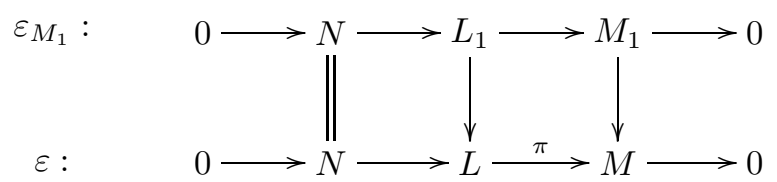

where $L_{1}$ is the pullback, and

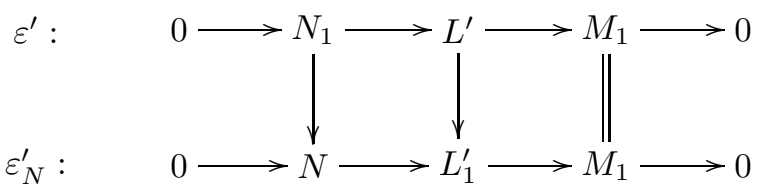

where $L_{1}^{\prime}$ is the pushout. It is clear that $\varepsilon, \varepsilon^{\prime}$ and $M_{1}, N_{1}$ induce the inclusions $L_{1} \subseteq L$ and $L^{\prime} \subseteq L_{1}^{\prime}$ and

$$
p_{0}: \operatorname{Ext}_{A}^{1}(M, N) \oplus \operatorname{Ext}_{A}^{1}\left(M_{1}, N_{1}\right) \rightarrow \operatorname{Ext}_{A}^{1}(M, N)
$$

is a projection. By a similar discussion as in [7, Lemma 2.4.2], we know that

$$
p\left(\phi^{-1}\left(\left(M_{1}, N_{1}\right)\right)\right)=\mathbb{P}\left(p_{0}\left(\operatorname{Ker}\left(\beta_{M_{1}, N_{1}}^{\prime}\right)\right)\right) .
$$

Moreover, by [8, Lemma 7], for fixed $\varepsilon \in p_{0}\left(\operatorname{Ker}\left(\beta_{M_{1}, N_{1}}^{\prime}\right)\right)$, let $\mathbb{P} \varepsilon$ be its orbit in $\mathbb{P}\left(p_{0}\left(\operatorname{Ker}\left(\beta_{M_{1}, N_{1}}^{\prime}\right)\right)\right)$. Then we have

$$
p^{-1}(\mathbb{P} \varepsilon) \cap \phi^{-1}\left(\left(M_{1}, N_{1}\right)\right) \cong \operatorname{Hom}\left(M_{1}, N / N_{1}\right) .
$$

Using Proposition 1.1] we get

$$
\chi\left(\phi^{-1}\left(\left(M_{1}, N_{1}\right)\right)\right)=\chi\left(\mathbb{P}\left(p_{0}\left(\operatorname{Ker}\left(\beta_{M_{1}, N_{1}}^{\prime}\right)\right)\right)=\operatorname{dim}_{\mathbb{C}} p_{0}\left(\operatorname{Ker}\left(\beta_{M_{1}, N_{1}}^{\prime}\right)\right) .\right.
$$


In the same way, we consider the projection

$$
\varphi: \mathbb{P} E F_{\underline{e}}^{g}(N, M) \rightarrow \bigcup_{\underline{e}_{1}+\underline{e}_{2}=\underline{e}} G r_{\underline{e}_{1}}(M) \times G r_{\underline{e}_{2}}(N) .
$$

Then

$$
\chi\left(\varphi^{-1}\left(\left(M_{1}, N_{1}\right)\right)\right)=\operatorname{dim}_{\mathbb{C}} \operatorname{Ext}_{A}^{1}(N, M) \cap \operatorname{Im} \beta_{N_{1}, M_{1}} .
$$

Now, depending on the fact that $\mathcal{C}(\mathcal{S})$ is of Ext-symmetry, we have

$$
\operatorname{dim}_{\mathbb{C}} p_{0}\left(\operatorname{Ker}\left(\beta_{M_{1}, N_{1}}^{\prime}\right)\right)+\operatorname{dim}_{\mathbb{C}} \operatorname{Ext}_{A}^{1}(N, M) \cap \operatorname{Im} \beta_{N_{1}, M_{1}}=\operatorname{dim}_{\mathbb{C}} \operatorname{Ext}_{A}^{1}(M, N) .
$$

Using Proposition 1.1 again, we complete the proof of the theorem.

\section{The MUltiplication FORMUla}

The formula in the last section is not so 'symmetric' as the Geiss-Leclerc-Schröer formula. In order to overcome this difficulty, we should consider flags of composition series instead of Grassmannians of submodules as in 7. In this section, we prove a multiplication formula as an analog of the Geiss-Leclerc-Schröer formula in [7].

Let $A=\mathbb{C} Q / \mathcal{I}$ be an algebra associated to a finite and connected quiver $Q$ and let $\mathcal{S}=\left\{S_{1}, \cdots, S_{n}\right\}$ be a finite set of finite-dimensional simple $A$-modules. Let $\mathcal{C}(\mathcal{S})$ be a full subcategory of Ext-symmetry of $\bmod (A)$ associated to $\mathcal{S}$.

Let $A_{\underline{d}}$ be the constructible subset of $\mathbb{E}_{\underline{d}}(A)$ consisting of $A$-modules in $\mathcal{C}(\mathcal{S})$ with dimension vector $\underline{d}$. Let $\mathcal{X}$ be the set of pairs $(\mathbf{j}, \mathbf{c})$ where $\mathbf{c}=\left(c_{1}, \cdots, c_{m}\right) \in\{0,1\}^{m}$ and $\mathbf{j}=\left(j_{1}, \cdots, j_{m}\right)$ is a sequence of integers such that $S_{j_{k}} \in \mathcal{S}$ for $1 \leq k \leq m$. Given $x \in A_{\underline{d}}$ and $(\mathbf{j}, \mathbf{c}) \in \mathcal{X}$, we define an $x$-stable flag of type $(\mathbf{j}, \mathbf{c})$ as a composition series of $x$,

$$
\mathfrak{f}_{x}=\left(V=\left(\mathbb{C}^{\underline{d}}, x\right) \supseteq V^{1} \supseteq \cdots \supseteq V^{m}=0\right),
$$

of $A$-submodules of $V$ such that $\left|V^{k-1} / V^{k}\right|=c_{k} S_{j_{k}}$, where $S_{j_{k}}$ is the simple module in $\mathcal{S}$. Let $\Phi_{\mathbf{j}, \mathbf{c}, x}$ be the variety of $x$-stable flags of type $(\mathbf{j}, \mathbf{c})$. We simply write $\Phi_{\mathbf{j}, x}$ when $\mathbf{c}=(1,1, \cdots, 1)$. Define

$$
\Phi_{\mathbf{j}}\left(A_{\underline{d}}\right)=\left\{(x, \mathfrak{f}) \mid x \in A_{\underline{d}}, \mathfrak{f} \in \Phi_{\mathbf{j}, x}\right\} .
$$

As in Proposition 1.3, we consider a projection: $p: \Phi_{\mathbf{j}}\left(A_{\underline{d}}\right) \rightarrow A_{\underline{d}}$. The function $p_{*}\left(1_{\Phi_{\mathbf{j}}\left(A_{\underline{d}}\right)}\right)$ is constructible by Theorem 1.2

Proposition 2.1. For any type $\boldsymbol{j}$, the function $A_{\underline{d}} \rightarrow \mathbb{C}$ mapping $x$ to $\chi\left(\Phi_{j, x}\right)$ is constructible.

Let $d_{\mathbf{j}, \mathbf{c}}: \mathbb{E}_{\underline{d}}(A) \rightarrow \mathbb{C}$ be the function defined by $d_{\mathbf{j}, \mathbf{c}}(x)=\chi\left(\Phi_{\mathbf{j}, \mathbf{c}, x}\right)$ for $x \in$ $\mathbb{E}_{\underline{d}}(A)$. It is a constructible function as in Proposition 2.1. We simply write $d_{\mathbf{j}}$ if $\mathbf{c}=(1, \cdots, 1)$. Define $\mathcal{M}(\underline{d})$ to be the vector space spanned by $d_{\mathbf{j}}$. For fixed $A_{\underline{d}}$, there are finitely many types $\mathbf{j}$ such that $\Phi_{\mathbf{j}}\left(A_{\underline{d}}\right)$ is not empty. Hence, there exists a finite subset $S(\underline{d})$ of $A_{\underline{d}}$ such that

$$
A_{\underline{d}}=\bigcup_{M \in S(\underline{d})}\langle M\rangle,
$$

where $\langle M\rangle=\left\{M^{\prime} \in A_{\underline{d}} \mid \chi\left(\Phi_{\mathbf{j}, M^{\prime}}\right)=\chi\left(\Phi_{\mathbf{j}, M}\right)\right.$ for any type $\left.\mathbf{j}\right\}$.

For any $M \in A_{\underline{d}}$, we define the evaluation form $\delta_{M}: \mathcal{M}(\underline{d}) \rightarrow \mathbb{C}$ mapping a constructible function $f \in \mathcal{M}(\underline{d})$ to $f(M)$. We have

$$
\langle M\rangle=\left\{M^{\prime} \in A_{\underline{d}} \mid \delta_{M^{\prime}}=\delta_{M}\right\} .
$$

Lemma 2.2. For $M, N \in \mathcal{C}(\mathcal{S})$, we have $\delta_{M \oplus N}=\delta_{M} \cdot \delta_{N}$. 
The lemma is equivalent to showing that

$$
\chi\left(\Phi_{\mathbf{j}, M \oplus N}\right)=\sum_{\mathbf{c}^{\prime}+\mathbf{c}^{\prime \prime} \sim 1} \chi\left(\Phi_{\mathbf{j}, \mathbf{c}^{\prime}, M}\right) \cdot \chi\left(\Phi_{\mathbf{j}, \mathbf{c}^{\prime \prime}, N}\right) .
$$

Here, $\mathbf{c}^{\prime}+\mathbf{c}^{\prime \prime} \sim 1$ means that $c_{k}^{\prime}+c_{k}^{\prime \prime}=1$ for $k=1, \cdots, m$. The proof of the lemma depends on the fact that under the action of $\mathbb{C}^{*}, \Phi_{\mathbf{j}, M \oplus N}$ and its stable subset have the same Euler characteristic. We refer to [6] for details.

The following formula is just the multiplication formula in [7, Theorem 1] when $A$ is a preprojective algebra and $\mathcal{S}$ is the set of all simple $A$-modules.

Theorem 2.3. With the above notation, for $M, N \in \mathcal{C}(\mathcal{S})$, we have

$$
\chi\left(\mathbb{P E x t}_{A}^{1}(M, N)\right) \delta_{M \oplus N}=\sum_{L \in S(\underline{e})}\left(\chi\left(\operatorname{PExt}_{A}^{1}(M, N)_{\langle L\rangle}\right)+\chi\left(\operatorname{PExt}_{A}^{1}(N, M)_{\langle L\rangle}\right)\right) \delta_{L},
$$

where $\underline{e}=\underline{\operatorname{dim}} M+\underline{\operatorname{dim}} N$.

In the proof of Theorem 1.5. a key point is to consider the linear maps $\beta_{M_{1}, N_{1}}$ and $\beta_{M_{1}, N_{1}}^{\prime}$ dual to each other by the property of Ext-symmetry. Now we extend this idea to the present situation as in [7]. Let

$$
\mathfrak{f}_{M}=\left(M=M_{0} \supseteq M_{1} \supseteq \cdots \supseteq M_{m}=0\right)
$$

be a flag of type $\left(\mathbf{j}, \mathbf{c}^{\prime}\right)$ and let

$$
\mathfrak{f}_{N}=\left(N=N_{0} \supseteq N_{1} \supseteq \cdots \supseteq N_{m}=0\right)
$$

be a flag of type $\left(\mathbf{j}, \mathbf{c}^{\prime \prime}\right)$ such that $c_{k}^{\prime}+c_{k}^{\prime \prime}=1$ for $k=1, \cdots, m$. We write $\mathbf{c}^{\prime}+\mathbf{c}^{\prime \prime} \sim 1$. For $k=1, \cdots, m$, let $\iota_{M, k}$ and $\iota_{N, k}$ be the inclusion maps $M_{k} \rightarrow M_{k-1}$ and $N_{k} \rightarrow N_{k-1}$, respectively. Define [7, Section 2]

$$
\beta_{\mathbf{j}, \mathbf{c}^{\prime}, \mathbf{c}^{\prime \prime}, \mathfrak{f}_{M}, \mathfrak{f}_{N}}: \bigoplus_{k=0}^{m-2} \operatorname{Ext}_{A}^{1}\left(N_{k}, M_{k+1}\right) \rightarrow \bigoplus_{k=0}^{m-2} \operatorname{Ext}_{A}^{1}\left(N_{k}, M_{k}\right)
$$

by the following map:

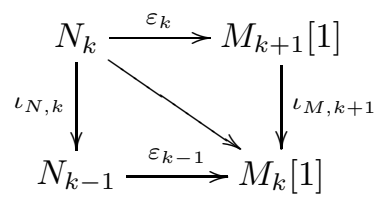

satisfying

$$
\beta_{\mathbf{j}, \mathbf{c}^{\prime}, \mathbf{c}^{\prime \prime}, \mathfrak{f}_{M}, \mathfrak{f}_{N}}\left(\varepsilon_{0}, \cdots, \varepsilon_{m-2}\right)=\iota_{M, 1} \circ \varepsilon_{0}+\sum_{k=1}^{m-2}\left(\iota_{M, k+1} \circ \varepsilon_{k}-\varepsilon_{k-1} \circ \iota_{N, k}\right) .
$$

Depending on the fact that $\mathcal{C}(\mathcal{S})$ is of Ext-symmetry, we can write its dual

$$
\beta_{\mathbf{j}, \mathbf{c}^{\prime}, \mathbf{c}^{\prime \prime}, \mathfrak{f}_{M}, \mathfrak{f}_{N}}^{\prime}: \bigoplus_{k=0}^{m-2} \operatorname{Ext}_{A}^{1}\left(M_{k}, N_{k}\right) \rightarrow \bigoplus_{k=0}^{m-2} \operatorname{Ext}_{A}^{1}\left(M_{k+1}, N_{k}\right)
$$

by the following map:

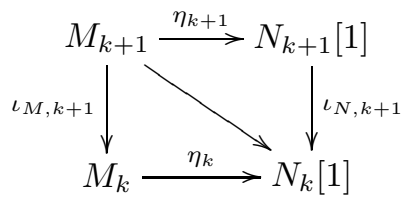


satisfying

$$
\beta_{\mathbf{j}, \mathbf{c}^{\prime}, \mathbf{c}^{\prime \prime}, \mathfrak{f}_{M}, \mathfrak{f}_{N}}^{\prime}\left(\eta_{0}, \cdots, \eta_{m-2}\right)=\sum_{k=0}^{m-3}\left(\eta_{k} \circ \iota_{M, k+1}-\iota_{N, k+1} \circ \eta_{k+1}\right)+\eta_{m-2} \circ \iota_{M, m-1} .
$$

Now, we prove Theorem 2.3 .

Proof. Define

$$
E F_{\mathbf{j}}(M, N)=\left\{(\varepsilon, \mathfrak{f}) \mid \varepsilon \in \operatorname{Ext}_{A}^{1}(M, N)_{L}, L \in A_{\underline{e}}, \mathfrak{f} \in \Phi_{\mathbf{j}, L}\right\}
$$

The action of $\mathbb{C}^{*}$ on $\operatorname{Ext}_{A}^{1}(M, N)$ induces an action on $E F_{\mathbf{j}}(M, N)$. The orbit space under the action of $\mathbb{C}^{*}$ is denoted by $\mathbb{P} E F_{\mathbf{j}}(M, N)$, and the orbit of $(\varepsilon, \mathfrak{f})$ is denoted by $\mathbb{P}(\varepsilon, \mathfrak{f})$. We have the natural projection

$$
p: \mathbb{P E F} F_{\mathbf{j}}(M, N) \rightarrow \operatorname{PExt}_{A}^{1}(M, N) .
$$

The fibre for any $\mathbb{P} \varepsilon \in \mathbb{P E x t}_{A}^{1}(M, N)_{L}$ is isomorphic to $\Phi_{\mathbf{j}, L}$. By Theorem 1.1, we have

$$
\chi\left(\mathbb{P E} F_{\mathbf{j}}(M, N)\right)=\sum_{L \in S(\underline{e})} \chi\left(\mathbb{P E x t}{ }_{A}^{1}(M, N)\langle L\rangle\right) \chi\left(\Phi_{\mathbf{j}, L}\right) .
$$

We also have the natural morphism

$$
\phi: \mathbb{P} E F_{\mathbf{j}}(M, N) \rightarrow \bigcup_{\mathbf{c}^{\prime}+\mathbf{c}^{\prime \prime} \sim 1} \Phi_{\mathbf{j}, \mathbf{c}^{\prime}, M} \times \Phi_{\mathbf{j}, \mathbf{c}^{\prime \prime}, N}
$$

mapping $\mathbb{P}(\varepsilon, \mathfrak{f})$ to $\left(\mathfrak{f}_{M}, \mathfrak{f}_{N}\right)$, where $\left(\mathfrak{f}_{M}, \mathfrak{f}_{N}\right)$ is naturally induced by $\varepsilon$ and $\mathfrak{f}$ and $t .(\varepsilon, \mathfrak{f})$ induces the same $\left(\mathfrak{f}_{M}, \mathfrak{f}_{N}\right)$ for any $t \in \mathbb{C}^{*}$. By [7, Lemma 2.4.2], we know that

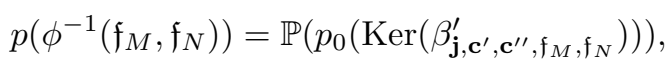

where $p_{0}: \bigoplus_{k=0}^{m-2} \operatorname{Ext}_{A}^{1}\left(M_{k}, N_{k}\right) \rightarrow \operatorname{Ext}_{A}^{1}(M, N)$ is a projection. On the other hand, by [8, Lemma 7], the morphism

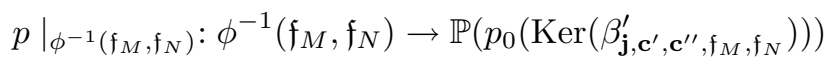

has fibres isomorphic to an affine space. Hence, by Theorem 1.1, we have

$$
\chi\left(\phi^{-1}\left(\mathfrak{f}_{M}, \mathfrak{f}_{N}\right)\right)=\chi\left(\mathbb{P}\left(p_{0}\left(\operatorname{Ker}\left(\beta_{\mathbf{j}, \mathbf{c}^{\prime}, \mathbf{c}^{\prime \prime}, \mathfrak{f}_{M}, \mathfrak{f}_{N}}^{\prime}\right)\right)\right) .\right.
$$

Dually, we define

$$
E F_{\mathbf{j}}(N, M)=\left\{(\varepsilon, \mathfrak{f}) \mid \varepsilon \in \operatorname{Ext}_{A}^{1}(N, M)_{L}, L \in A_{\underline{e}}, \mathfrak{f} \in \Phi_{\mathbf{j}, L}\right\} .
$$

The orbit space under $\mathbb{C}^{*}$-action is denoted by $\mathbb{P} E F_{\mathbf{j}}(N, M)$. We have the natural projection

$$
q: \mathbb{P} E F_{\mathbf{j}}(N, M) \rightarrow \mathbb{P E x t}_{A}^{1}(N, M) .
$$

The fibre for any $\mathbb{P} \varepsilon \in \mathbb{P E x t}_{A}^{1}(N, M)_{L}$ is isomorphic to $\Phi_{\mathbf{j}, L}$. By Theorem 1.1, we have

$$
\chi\left(\mathbb{P E F} F_{\mathbf{j}}(N, M)\right)=\sum_{L \in S(\underline{e})} \chi\left(\operatorname{PExt}_{A}^{1}(N, M)_{\langle L\rangle}\right) \chi\left(\Phi_{\mathbf{j}, L}\right) .
$$

As in the proof of Theorem 1.5, there is a natural morphism

$$
\varphi_{0}: E F_{\mathbf{j}}(N, M) \rightarrow \bigcup_{\mathbf{c}^{\prime}+\mathbf{c}^{\prime \prime} \sim 1} \Phi_{\mathbf{j}, \mathbf{c}^{\prime}, M} \times \Phi_{\mathbf{j}, \mathbf{c}^{\prime \prime}, N}
$$

such that

$$
\varphi_{0}((\varepsilon, \mathfrak{f}))=\varphi_{0}(t .(\varepsilon, \mathfrak{f}))
$$


for any $(\varepsilon, \mathfrak{f}) \in E F_{\mathbf{j}}(N, M)$ and $t \in \mathbb{C}^{*}$. Hence, we have the morphism

$$
\varphi: \mathbb{P E F} \mathbf{j}(N, M) \rightarrow \bigcup_{\mathbf{c}^{\prime}+\mathbf{c}^{\prime \prime} \sim 1} \Phi_{\mathbf{j}, \mathbf{c}^{\prime}, M} \times \Phi_{\mathbf{j}, \mathbf{c}^{\prime \prime}, N} .
$$

By [7, Lemma 2.4.3], we know that

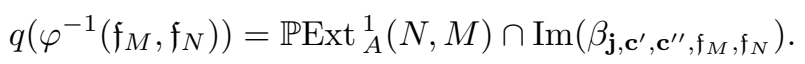

Similar to the above dual situation, by [8, Lemma 7], the morphism

$$
\left.q\right|_{\varphi^{-1}\left(\mathfrak{f}_{M}, \mathfrak{f}_{N}\right)}: \varphi^{-1}\left(\mathfrak{f}_{M}, \mathfrak{f}_{N}\right) \rightarrow \mathbb{P E x t}{ }_{A}^{1}(N, M) \cap \operatorname{Im}\left(\beta_{\left.\mathbf{j}, \mathbf{c}^{\prime}, \mathbf{c}^{\prime \prime}, \mathfrak{f}_{M}, \mathfrak{f}_{N}\right)}\right.
$$

has fibres isomorphic to an affine space. Hence, by Proposition 1.1, we have

$$
\chi\left(\varphi^{-1}\left(\mathfrak{f}_{M}, \mathfrak{f}_{N}\right)\right)=\chi\left(\mathbb{P E x t}_{A}^{1}(N, M) \cap \operatorname{Im}\left(\beta_{\mathbf{j}, \mathbf{c}^{\prime}, \mathbf{c}^{\prime \prime}, \mathfrak{f}_{M}, \mathfrak{f}_{N}}\right)\right) .
$$

However, since $\beta_{\mathbf{j}, \mathbf{c}^{\prime}, \mathbf{c}^{\prime \prime}, \mathfrak{f}_{M}, \mathfrak{f}_{N}}$ and $\beta_{\mathbf{j}, \mathbf{c}^{\prime}, \mathbf{c}^{\prime \prime}, \mathfrak{f}_{M}, \mathfrak{f}_{N}}$ are dual to each other, we have

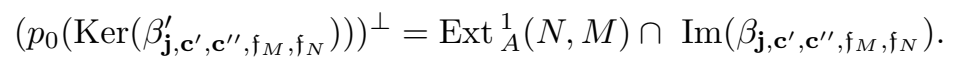

Thus we have

$$
\begin{aligned}
& \chi\left(\mathbb{P}\left(p_{0}\left(\operatorname{Ker}\left(\beta_{\mathbf{j}, \mathbf{c}^{\prime}, \mathbf{c}^{\prime \prime}, \mathfrak{f}_{M}, \mathfrak{f}_{N}}\right)\right)\right)\right)+\chi\left(\mathbb{P E x t}_{A}^{1}(N, M) \cap \operatorname{Im}\left(\beta_{\left.\left.\mathbf{j}, \mathbf{c}^{\prime}, \mathbf{c}^{\prime \prime}, \mathfrak{f}_{M}, \mathfrak{f}_{N}\right)\right)}\right)\right. \\
= & \operatorname{dim}_{\mathbb{C}} \operatorname{Ext}_{A}^{1}(M, N) .
\end{aligned}
$$

Therefore, using Proposition 1.1, we obtain

$$
\mathbb{P} E F_{\mathbf{j}}(M, N)+\mathbb{P} E F_{\mathbf{j}}(N, M)=\operatorname{dim}_{\mathbb{C}} \operatorname{Ext}^{1}(M, N) \cdot \sum_{\mathbf{c}^{\prime}+\mathbf{c}^{\prime \prime} \sim 1} \chi\left(\Phi_{\mathbf{j}, \mathbf{c}^{\prime}, M}\right) \cdot \chi\left(\Phi_{\mathbf{j}, \mathbf{c}^{\prime \prime}, N}\right) .
$$

Now, we have obtained the identity

$$
\begin{aligned}
& \operatorname{dim}_{\mathbb{C}} \operatorname{Ext}^{1}(M, N) \cdot \sum_{\mathbf{c}^{\prime}+\mathbf{c}^{\prime \prime} \sim 1} \chi\left(\Phi_{\mathbf{j}, \mathbf{c}^{\prime}, M}\right) \cdot \chi\left(\Phi_{\mathbf{j}, \mathbf{c}^{\prime \prime}, N}\right) \\
& =\sum_{L \in S(\underline{e})} \chi\left(\mathbb{P E x t}_{A}^{1}(M, N)_{\langle L\rangle}\right) \chi\left(\Phi_{\mathbf{j}, L}\right)+\sum_{L \in S(\underline{e})} \chi\left(\mathbb{P E x t}{ }_{A}^{1}(N, M)_{\langle L\rangle}\right) \chi\left(\Phi_{\mathbf{j}, L}\right)
\end{aligned}
$$

for any type $\mathbf{j}$. Using Lemma 2.2 and Proposition 1.1, we finish the proof of Theorem 2.3 .

\section{EXAmples}

In this section, we give some examples of module subcategories of Ext-symmetry. (I) Let $A$ be a preprojective algebra associated to a connected quiver $Q$ without loops. Let $\mathcal{S}$ be the set of all simple $A$-modules. Then $\mathcal{C}(\mathcal{S})$ is of Ext-symmetry [7, Theorem 3].

(II) Let $A=\mathbb{C} Q /\left\langle\alpha \alpha^{*}-\alpha^{*} \alpha\right\rangle$ be an associative algebra associated to the following quiver:

$$
Q:=\alpha C^{0} \alpha^{*}
$$

Let $M=\left(\mathbb{C}^{m}, X_{\alpha}, X_{\alpha^{*}}\right)$ and $N=\left(\mathbb{C}^{n}, Y_{\alpha}, Y_{\alpha^{*}}\right)$ be two finite-dimensional $A$ modules. Following the characterization of $\operatorname{Ext}_{A}^{1}(M, N)$ in Section 1.3, we consider the following isomorphism between complexes (see [3, Lemma 1] or [7, Section 8.2]):

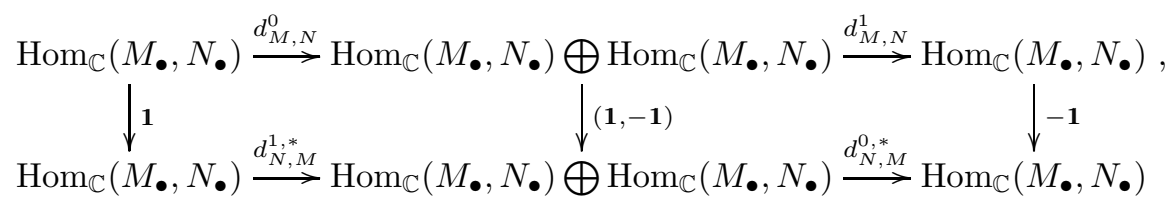


where $M_{\bullet}=\mathbb{C}^{m}, N_{\bullet}=\mathbb{C}^{n}$. Here, we define

$$
\begin{aligned}
d_{M, N}^{0}(A) & =\left(Y_{\alpha} A-A X_{\alpha}, Y_{\alpha^{*}} A-A X_{\alpha^{*}}\right), d_{M, N}^{1}\left(B, B^{*}\right) \\
& =Y_{\alpha^{*}} B+B^{*} X_{\alpha}-Y_{\alpha} B^{*}-B X_{\alpha^{*}} \\
d_{N, M}^{0, *}\left(B, B^{*}\right) & =B X_{\alpha^{*}}+B^{*} X_{\alpha}-Y_{\alpha^{*}} B-Y_{\alpha} B^{*}, d_{N, M}^{1, *}(A) \\
& =\left(Y_{\alpha} A-A X_{\alpha},-Y_{\alpha^{*}} A+A X_{\alpha^{*}}\right)
\end{aligned}
$$

for any $n \times m$ matrices $A, B$ and $B^{*}$. The second complex is dual to the complex

$$
\operatorname{Hom}_{\mathbb{C}}\left(N_{\bullet}, M_{\bullet}\right) \stackrel{d_{N, M}^{0}}{\longrightarrow} \operatorname{Hom}_{\mathbb{C}}\left(N_{\bullet}, M_{\bullet}\right) \bigoplus \operatorname{Hom}_{\mathbb{C}}\left(N_{\bullet}, M_{\bullet}\right) \stackrel{d_{N, M}^{1}}{\longrightarrow} \operatorname{Hom}_{\mathbb{C}}\left(N_{\bullet}, M_{\bullet}\right)
$$

with respect to the nondegenerate bilinear form

$$
\Phi: \operatorname{Hom}_{\mathbb{C}}\left(N_{\bullet}, M_{\bullet}\right) \times \operatorname{Hom}_{\mathbb{C}}\left(N_{\bullet}, M_{\bullet}\right) \rightarrow \mathbb{C}
$$

mapping $(X, Y)$ to $\operatorname{tr}(X Y)$. As in Section 1.3, we have functorially

$\operatorname{Ext}_{A}^{1}(M, N)=\operatorname{Ker}\left(d_{M, N}^{1}\right) / \operatorname{Im}\left(d_{M, N}^{0}\right)$ and $\operatorname{DExt}_{A}^{1}(N, M)=\operatorname{Ker}\left(d_{N, M}^{0, *}\right) / \operatorname{Im}\left(d_{N, M}^{1, *}\right)$.

Hence, we have a bifunctorial isomorphism:

$$
\operatorname{Ext}_{A}^{1}(M, N) \cong \operatorname{DExt}_{A}^{1}(N, M) \text {. }
$$

(III) Deformed preprojective algebras were introduced by Crawley-Boevey and Holland in [4. Fix $\lambda=\left(\lambda_{i}\right)_{i \in Q_{0}}$ where $\lambda_{i} \in \mathbb{C}$. The deformed preprojective algebra of weight $\lambda$ is an associative algebra

$$
A(\lambda)=\mathbb{C} \bar{Q} /\left\langle\sum_{\alpha \in Q_{1}}\left(\alpha \alpha^{*}-\alpha^{*} \alpha\right)-\sum_{i \in Q_{0}} \lambda_{i} e_{i}\right\rangle,
$$

where $\bar{Q}=Q \cup Q^{*}$ is the double of a quiver $Q$ without loops. Let $M, N$ be finitedimensional $A$-modules. As in Section [1.3, we know $D(M, N)$ is just the kernel of the following linear map:

$$
\bigoplus_{\alpha \in \bar{Q}_{1}} \operatorname{Hom}_{\mathbb{C}}\left(M_{s(\alpha)}, N_{t(\alpha)}\right) \stackrel{d_{M, N}^{1}}{\longrightarrow} \bigoplus_{i \in Q_{0}} \operatorname{Hom}_{\mathbb{C}}\left(M_{i}, N_{i}\right),
$$

where $d_{M, N}^{1}$ maps $\left(f_{\alpha}\right)_{\alpha \in \bar{Q}_{1}}$ to $\left(g_{i}\right)_{i \in Q_{0}}$ such that

$$
g_{i}=\sum_{\alpha \in Q_{1}, s(\alpha)=i}\left(N_{\alpha^{*}} f_{\alpha}+f_{\alpha^{*}} M_{\alpha}\right)-\sum_{\alpha \in Q_{1}, t(\alpha)=i}\left(N_{\alpha} f_{\alpha^{*}}+f_{\alpha} M_{\alpha^{*}}\right) .
$$

In the same way as in [7, Section 8.2], we obtain a bifunctorial isomorphism

$$
\operatorname{Ext}_{A}^{1}(M, N) \cong \operatorname{DExt}_{A}^{1}(N, M) \text {. }
$$

(IV) It is easy to construct examples of module subcategories of Ext-symmetry over an algebra which is not of Ext-symmetry. Let $A=\mathbb{C} Q /\left\langle\beta \beta^{*}-\beta^{*} \beta\right\rangle$ be a quotient algebra associated to the quiver

$$
Q:=1 \stackrel{\alpha}{\longrightarrow} 2 \underset{\beta^{*}}{\stackrel{\beta}{\longrightarrow}} 3 .
$$

Let $S_{1}, S_{2}$ and $S_{3}$ be finite-dimensional simple $A$-modules associated to three vertices, respectively. Since $\operatorname{dim}_{\mathbb{C}} \operatorname{Ext}^{1}\left(S_{1}, S_{2}\right)=1$ and $\operatorname{Ext}^{1}\left(S_{2}, S_{1}\right)=0, A$ is not an algebra of Ext-symmetry. However, for $\mathcal{S}=\left\{S_{1}, S_{3}\right\}$ or $\left\{S_{2}, S_{3}\right\}, \mathcal{C}(\mathcal{S})$ is of Ext-symmetry. 


\section{ACKNOWLEDGMENTS}

We are grateful to the referee for many helpful comments. In particular, Section 3 was added following the comments. Furthermore, the second author would like to thank the Max Planck Institute for Mathematics in Bonn for a three-month research stay in 2008 .

\section{REFERENCES}

1. P. Caldero, F. Chapoton, Cluster algebras as Hall algebras of quiver representations, Comm. Math. Helv. 81 (2006), 595-616. MR2250855 (2008b:16015)

2. P. Caldero, B. Keller, From triangulated categories to cluster algebras, Inv. Math. 172 (2008), 169-211. MR2385670

3. W. Crawley-Boevey, On the exceptional fibres of Kleinian singularities, Amer. J. Math. 122 (2000), no. 5, 1027-1037. MR 1781930 (2001f:14009)

4. W. Crawley-Boevey, M. Holland, Noncommutative deformations of Kleinian singularities, Duke Math. J. 92 (1998), no. 3, 605-635. MR1620538 (99f:14003)

5. A. Dimca, Sheaves in topology. Universitext. Springer-Verlag, Berlin, 2004. MR2050072 (2005j:55002)

6. M. Ding, J. Xiao, F. Xu, Realizing enveloping algebras via varieties of modules, arXiv:math/0604560.

7. C. Geiss, B. Leclerc, J. Schröer, Semicanonical bases and preprojective algebras, II: A multiplication formula, Compositio Mathematica 143 (2007), 1313-1334. MR2360317 (2009b:17031)

8. A. Hubery, Acyclic cluster algebras via Ringel-Hall algebras, preprint.

9. D. Joyce, Constructible functions on Artin stacks, J. London Math. Soc. (2) 74 (2006), 583606. MR2286434 (2008b:14001)

10. R. D. MacPherson, Chern classes for singular algebraic varieties, Ann. of Math. (2) 100 (1974), 423-432. MR0361141 (50:13587)

11. Ch. Riedtmann, Lie algebras generated by indecomposables, J. Algebra 170 (1994), 526-546. MR.1302854 (96e:16013)

12. C. M. Ringel, The preprojective algebra of a quiver. In: Algebras and modules II (Geiranger, 1996), 467-480, CMS Conf. Proc. 24, Amer. Math. Soc., Providence, RI, 1998. MR 1648647 (99i:16031)

Department of Mathematics, Tsinghua University, Beijing 100084, People's Republic OF CHINA

E-mail address: jxiao@math.tsinghua.edu.cn

Department of Mathematics, Tsinghua University, Beijing 100084, People's Republic OF CHINA

E-mail address: fanxu@mail.tsinghua.edu.cn 